Cahiers $d u$ MONDE RUSSE

\section{Cahiers du monde russe}

Russie - Empire russe - Union soviétique et États indépendants

$58 / 4 \mid 2017$

Varia

\title{
Geneviève Piron, Lada Umstätter, éds., L'Utopie au quotidien : La vie ordinaire en URSS, 1953-1985
}

\section{Olga Medvedkova}

\section{(2) OpenEdition}

\section{Journals}

Édition électronique

URL : http://journals.openedition.org/monderusse/10181

DOI : 10.4000/monderusse. 10181

ISSN : $1777-5388$

\section{Éditeur}

Éditions de l'EHESS

\section{Édition imprimée}

Date de publication : 1 octobre 2017

Pagination : 735-737

ISBN : 978-2-7132-2698-4

ISSN : $1252-6576$

\section{Référence électronique}

Olga Medvedkova, « Geneviève Piron, Lada Umstätter, éds., L'Utopie au quotidien : La vie ordinaire en URSS, 1953-1985 », Cahiers du monde russe [En ligne], 58/4 | 2017, mis en ligne le 01 octobre 2017, consulté le 05 janvier 2021. URL : http://journals.openedition.org/monderusse/10181 ; DOI : https:// doi.org/10.4000/monderusse.10181 
Geneviève PIRON, Lada UMSTÄTTER, éds.

\section{L'Utopie au quotidien}

\section{La vie ordinaire en URSS, 1953-1985}

Paris - Lausanne : Noir sur blanc, 2017

À son retour d'URSS en 1936, André Gide est à la fois aux anges et aux enfers. Aux anges, car il a vu une belle humanité : naturelle, vigoureuse. Une humanité jeune, en maillot, qui court sous le soleil. Il prend donc « un bain d'humanité ». Il fait connaissance, envahi par la chaleur de son regard. Il n'aime pas les paysages ; il se moque des machines; en voyageant c'est l'homme qu'il cherche. Il le trouve donc, ou plutôt il trouve toute une humanité : un comme tous, tous comme un. Puis ça cloche et l'enfer commence, pratiquement parlant, en vue d'un magasin: une queue de deux ou trois cents personnes. Ils sont là, tendus devant la porte fermée : ils attendent l'arrivage de quelque chose, peut-être des coussins ; seuls les premiers en auront. Enfin, le magasin s'ouvre et l'esthète parisien voit l'objet soviétique. Il est rebutant. Tout est affreux. Une pensée absurde vrombit : c'est si vilain qu'on l'a sans doute fait exprès «pour modérer les appétits ». Il n'ose imaginer qu'une telle laideur puisse naître par mégarde. Il espère encore dénicher quelques souvenirs, mais non, impossible! Si au moins c'était prolétaire, mais tout est si «bêtement bourgeois, petit-bourgeois ». Et puis c'est partout pareil. Le nivellement social, c'est bien, c'est ce qu'on veut. Mais que faire de l'uniformité ? De l'aplatissement, de la raréfaction du monde matériel, qui finit par trahir l'uniformisation de l'humain.

Le livre L'Utopie au quotidien : la vie ordinaire en URSS qui vient de paraître aux éditions Noir sur Blanc introduit cet « objet soviétique » qui naguère rebuta Gide. La période que ce livre couvre, plus récente, est bien définie : à partir de la mort de Stalin en 1953 et jusqu'à la perestroïka qui s'annonce en 1985. La première date, celle de la déstalinisation et du dégel qui fut de courte durée, ne bouleverse guère le fonctionnement des choses dans la vie quotidienne sur l'immense territoire de l'URSS. La vision de Gide nous est toujours utile et même plus qu'utile, car cette vision désamorce non seulement toute nostalgie, mais encore tout sentiment de similitude. «Oh, 
ma grand-mère avait la même lampe. » Oui, mais la voisine d'en face en avait une autre. Et celle d'au-dessus une autre encore. Alors que cette lampe soviétique, tout le pays l'avait, depuis les steppes jusqu'au cercle polaire. Ce n'est donc jamais, malgré la ressemblance possible, le même objet. Ce n'est même pas un objet, dans son sens phénoménal (l'une ou l'autre incarnation de l'idée lampe ou de l'idée coussin, de telle ou telle forme ou de telle ou telle couleur), c'est l'idée de l'objet, sa définition au dictionnaire. Coussin: sorte de sac cousu de tous les côtés. Nous sommes dans l'univers des noumènes, dans le royaume de l'utopie. Le magasin soviétique est un dépôt d'idées platoniciennes. On y guette et parfois obtient LE coussin, LA lampe. Lénine ne fut-il pas un vaillant lecteur de Thomas More? "Les vêtements ont la même forme pour tous les habitants de l'île ; cette forme est invariable, elle distingue seulement l'homme de la femme, le célibat du mariage. »

Quant à la seconde date, 1985, c'est celle d'un changement radical. L'URSS s'écroule, l'objet occidental, phénoménal envahit l'espace ex-soviétique. La page est tournée. Paraissent, rapidement, des études quasi archéologiques. Nombre de projets de recherche, d'ouvrages savants et de vulgarisation voient le jour ${ }^{1}$. Le livre dont nous parlons ici en fait partie ${ }^{2}$; c'est une façon de répertoire des objets soviétiques obéissant à la logique «platonicienne », si férocement dévoilée par Gide. L'ouvrage s'appuie sur la collection réunie par Lada Umstätter, directrice du musée des Beaux-Arts de La Chaux-de-Fonds, collection qui a été exposée au même musée en février-avril 2017.

Le livre n'est pourtant pas un catalogue, et heureusement. Car, tels quels, tout seuls, ces choses ne parlent sans doute qu'aux ex-Soviétiques. Aux yeux de l'humanité occidentale qui n'a pas enduré le totalitarisme de ce type, elles restent muettes. Que signifie par exemple ce verre à facettes, à moitié rempli de graines de tournesol, qui figure sur la couverture ? Et pourtant c'est toute une histoire. Sans la connaître, sans savoir que, depuis la révolution prolétaire, la population paysanne envahit les villes, l'habitude paysanne de grignoter ces graines en crachant l'écale par terre s'étend jusqu'aux populations urbaines et que, durant la période soviétique, les babouchkas devant les bouches de métro vendent ces graines au verre, on ne peut rien comprendre à cette image. Le défi du livre a été donc d'expliciter ces choses, de les forcer à trahir leur secret, à dire leur nature paradoxale.

Ce défi, les auteures le réussissent à merveille. Premièrement, en répartissant les objets en types et catégorie, en les classant par fonction ou (ce qui est plus important sous le régime de la pénurie) par mode d'obtention. Ainsi le lecteur peut-il s'imaginer naître, grandir, habiter, traverser la vie, avoir tout ce qu'il faut, fêter, travailler, etc. avec les Soviétiques. On visualise leurs jouets (quelques types approuvés), l'insigne des enfants d'Octobre (avec le petit portrait de Lenin enfant), le foulard des pionniers (servant à l'occasion de mouchoir), le diplôme d'honneur avec les portraits de Lenin et de Stalin (1955) et sans ces derniers (1959, après le $\mathrm{XX}^{\mathrm{e}}$ congrès du Parti), le collant (en gros coton, toujours rapiécé), le collier (nécessairement d'ambre), le préservatif (introuvable), la boîte de chocolats (distribuée à l'occasion des fêtes) ou de pâtes de fruits (cadeau des syndicats), le vase en cristal de Bohème (cher, chic, symbole de réussite), un rouleau de papier hygiénique (qui 
disparaît régulièrement du commerce, poussant les peuples de l'URSS à employer le papier journal à la place). Tout le monde les possède ou les désire. Dans toutes les maisons il y a LA même théière à tulipes et LE même œuvre complet de Pouchkine à couverture grise. Les femmes se parfument au même Moscou rouge et, dans les années 1960, contemplent au mur le même portrait d'Hemingway.

Deuxièmement, les reproductions de ces objets-idées s'accompagnent dans le livre de textes historiques et d'une anthologie de fragments littéraires, écrits, choisis et traduits du russe par Geneviève Piron. Parmi les auteurs : Aleškovskij, Nekrasov, Gorenstein, Sokolov, Dovlatov, Iskander, Sorokin et tant d'autres. Une vaste collection de photographies y ajoute du relief. Ces dernières sont réellement étonnantes. Elles appartiennent à cette heureuse période, quand la photographie est encore révélatrice de la vie. Ces photos de classe si exactement pareilles qu'on pense se reconnaître dans chacune ! Ces cuisines, ces parcs de culture, ces datchas, ces cafés où on ne sert que de la glace, ces mariages (mêmes robes et complets), ces vitrines avec comme seul produit trente bocaux du même jus de pomme. Et comme leitmotiv, de petites gens sous des portraits énormes, sous les lettres colossales PCUS sur les toits, de petites gens renfrognées, aux pieds gonflés, sous les affiches gigantesques figurant leurs sosies idéaux, robustes, rayonnants.

Enfin, un glossaire très complet, y compris des termes de la langue de bois et du jargon (en russe et en traduction) est ajouté à la fin. On y apprend par exemple que les boîtes en fer pour des produits alimentaires d'une banalité rugissante n'ont été fabriquées que par une seule usine en Estonie, ce pourquoi tout le monde les achetait en y allant en vacances.

Le seul reproche que l'on oserait faire à ce livre, à cette triste et intelligente somme sur une utopie incarnée, puis disparue, est que l'objet polygraphique (le livre dans sa matérialité) est beau. Sans doute trop, vu ce dont il traite.

1 - Citons seulement : Sheila Fitzpatrick, Everyday Stalinism : Ordinary Life in Extraordinary Times, Soviet Russia in the 1930s, Oxford University Press, 1999 ; Larissa Zakharova, S'habiller à la soviétique : la mode et le dégel en URSS, P. : CNRS éditions, 2011. 2 - Le projet, dans sa partie historique et littéraire, a été porté par l'université de Genève et financé par le Fonds national suisse de la recherche scientifique.

Olga Medvedkova

Centre Jean Pépin (CNRS) 